\title{
Malus Law in terms of the geometric phase
}

\author{
Marzena Zdunek*, Władysław A. Woźniak \\ Institute of Physics, Wrocław University of Technology, Wybrzeże Wyspiańskiego 27, 50-370 Wrocław, Poland
}

Received October 30, 2013; accepted December 17, 2013; published December 31, 2013

\begin{abstract}
The aim of the present work is the description of the commonly known Malus law as the interference of two waves. We have proposed two cases: interference of two waves with different intensities and equal phases and interference of two waves with different phases and equal intensities. The first case can be realized by decomposition of the polarization state of the light incident on the analyzer into the base of linearly polarized waves. In the second case, the base of circularly polarized waves should be chosen. This last option allows applying a geometric phase concept to the Malus law.
\end{abstract}

The proof of the wave nature of light is a series of phenomena that can be explained only on the assumption that light is an electromagnetic wave. These phenomena include diffraction, interference and polarization. While diffraction and interference are often treated as linked together (in fact, diffraction is observed by the interference of waves, according to the Huygens - Fresnel principle) polarization is treated as a separate class, resulting not only from the treatment of light as a wave, but also the assumption that light is a transverse wave. The authors of this study believe that polarization can also be observed because of interference. In principle, waves interfere when they have the same states of polarization. If we want to cause interference of waves of different polarization states (for example eigenwaves of birefringent medium), we have to put the analyzer at the end of a measurement system. In our approach this element analyzes the light exactly by interference of its components. That is the description used in this paper with reference to the Malus law.

In the simplest version, for two ideal linear polarizers, Malus law states that the intensity of the light after passing through the setup (polarizer + analyzer) is proportional to the square cosine functions of the difference between the first polarizer $\left(\alpha_{P}\right)$ and analyzer $\left(\alpha_{A}\right)$ eigenvectors' azimuth angles:

$$
I_{\text {out }} \propto \cos ^{2}\left(\alpha_{A}-\alpha_{P}\right) .
$$

In the classic approach such a change of the outcoming light intensity is explained by the analyzers attenuation. On the other hand, the square cosine dependence of the output light beam intensity in Eq. (1) seems a typical

\footnotetext{
*E-mail: marzena.zdunek@pwr.wroc.pl
}

element of all interferometric formulas where the cosine function argument (here: $\alpha_{A}-\alpha_{P}$ ) serves as the phase difference between two interfering waves.

The phase difference between two interfering light beams may arise due to two reasons. Firstly, some optical elements can introduce their "own" phase shift as, for example, retardation plates used in anisotropic media optics. Secondly, the mutual orientation of optical elements can introduce another kind of phase, called geometrical. Since this cosine function argument in Malus law depends on mutual orientation of a polarizer and an analyzer, it can be considered to be a geometrical phase.

Let us describe the experiment in which the interference nature of Malus Law is easy to observe (Fig. 1). We have used the special construction called a spatial polarizer which allows generation of spatially varying polarization state distribution: linearly polarized lights with the continuous change of the azimuth angle. Placing an linear analyzer on such light's way, one can observe variable light intensity distribution in accordance with Eq. (1) (constant $\alpha_{\mathrm{A}}$ and spatially changeable $\alpha_{\mathrm{P}}$ ).
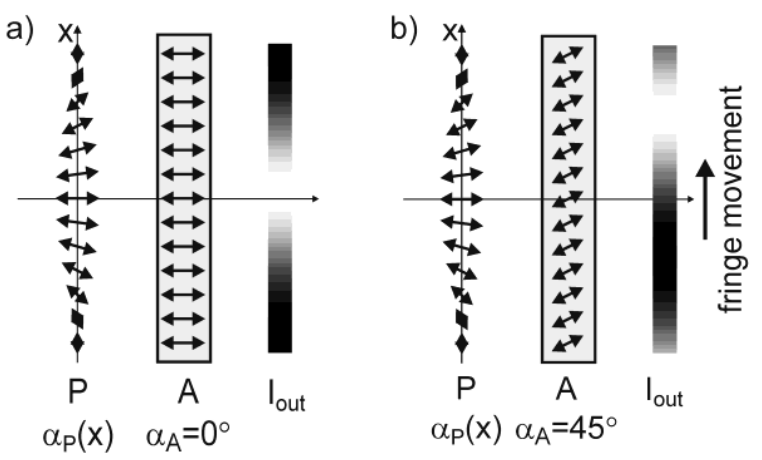

Fig. 1. Malus Law experiment with spatial polarizer: fringe movement caused by rotation of the analyzer similar to observed in interferometric experiments: a) analyzer with the azimuth angle $\alpha_{A}=0^{\circ}$ and b) analyzer rotated to azimuth angle $\alpha_{\mathrm{A}}=45^{\circ}$.

Due to the specific construction of our spatial polarizer, this intensity distribution forms a set of parallel fringes. Rotating the analyzer causes the shift of the fringes set the same shift, commonly observed in many interferometric experiments, which is always interpreted as a result of changes in the relative phase (phase shift) 
between two (or more) interfering waves. This might be a good starting point for supposing that the light intensity calculated with Malus Law can actually be treated as a result of interference thus making it possible to find the desired phase shift which we postulate as being geometrical.

Let us describe the above-mentioned Malus Law experiment formally using the Jones vector formalism. The Jones vector $\mathbf{E}_{\mathrm{P}}$ of the light emerging from the polarizer can be written as:

$$
\mathbf{E}_{P}=\left[\cos \alpha_{P}, \sin \alpha_{P}\right]
$$

This equation means, in fact, that vector $\mathbf{E}_{P}$ is represented in linear base consisting of two versors: $\hat{\mathbf{H}}=[1,0] \quad(x$-axis, horizontal) and $\hat{\mathbf{V}}=[0,1] \quad(y$-axis, vertical) and can be formally written as:

$$
\begin{aligned}
& \mathbf{E}_{P}=\left\langle\mathbf{E}_{P}, \hat{\mathbf{H}}\right\rangle \hat{\mathbf{H}}+\left\langle\mathbf{E}_{P}, \hat{\mathbf{V}}\right\rangle \hat{\mathbf{V}}= \\
& =\cos \alpha_{P} \hat{\mathbf{H}}+\sin \alpha_{P} \hat{\mathbf{V}} \equiv \mathbf{E}_{P H}+\mathbf{E}_{P V}
\end{aligned}
$$

where $\mathbf{E}_{P H}$ and $\mathbf{E}_{P V}$ denote the horizontal and vertical components of $\mathbf{E}_{P}$ vector, and $\left\langle\mathbf{E}_{P}, \hat{\mathbf{H}}\right\rangle$ denotes a scalar product of proper vectors. According to Malus Law the Jones vector $\mathbf{E}_{\text {out }}$ of the light outcoming from the analyzer should be written as:

$$
\mathbf{E}_{\text {out }}=\cos \left(\alpha_{A}-\alpha_{P}\right) \mathbf{A}
$$

and can be formally written as the projection of the Jones vector $\mathbf{E}_{P}$ onto the analyzer first "transmission" eigenvector A (the second eigenvector is fully attenuated):

$$
\mathbf{E}_{\text {out }}=\left\langle\mathbf{E}_{P}, \mathbf{A}\right\rangle \mathbf{A}
$$

Inserting Eq. (3) into Eq. (5) one can obtain the following formula:

$$
\begin{aligned}
& \mathbf{E}_{\text {out }}=\left(\cos \alpha_{P} \cos \alpha_{A}\right) \mathbf{A}+\left(\sin \alpha_{P} \sin \alpha_{A}\right) \mathbf{A} \equiv \\
& \equiv \mathbf{E}_{\text {out } H}+\mathbf{E}_{\text {out } V}
\end{aligned}
$$

where $\mathbf{E}_{\text {out } H}$ and $\mathbf{E}_{\text {out } V}$ denote the horizontal and vertical components of the $\mathbf{E}_{\text {out }}$.
The graphical representation of the $\mathbf{E}_{P}$ vector's decomposition as well as the projection of $\mathbf{E}_{P H}$ and $\mathbf{E}_{P V}$ components into vector $\mathbf{A}$ is presented in Fig. 2.

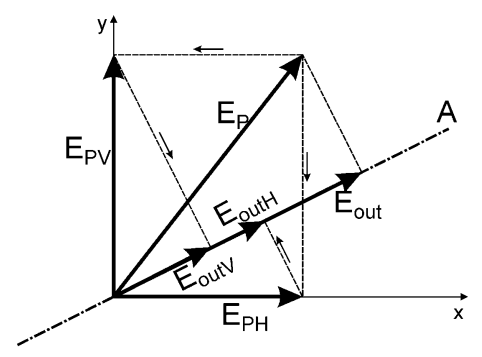

Fig. 2. The graphical representation of the $\mathbf{E}_{\mathrm{P}}$ vector's decomposition into vertical $\left(\mathbf{E}_{\mathrm{PV}}\right)$ and horizontal $\left(\mathbf{E}_{\mathrm{PH}}\right)$ linear components as well as the projection of $\mathbf{E}_{\mathrm{PH}}$ and $\mathbf{E}_{\mathrm{PV}}$ components into vector $\mathbf{A}$ to obtain the vector $\mathbf{E}_{\text {out }}$ of the light emerging from the analyzer.

Finally, we can conclude that Malus Law equation can be obtained as an effect of the interference of two linearly polarized waves ( $\mathbf{E}_{\text {out } H}$ and $\mathbf{E}_{\text {out } V}$ ) with the same polarization states (represented by vector $\mathbf{A}$ ), different amplitudes (dependent on the quantities $\alpha_{P}$ and $\alpha_{A}$ ) and the same phases.

It is easy to show that all linear polarization states can be represented also as a combinations of two circularly polarized states with opposite circularity and the same excitation coefficients. The azimuth angle of the decomposed linear state will depend on the mutual phase difference between circular states - the quantity we are, after all, looking for. As a consequence, finally we have proposed to choose as the desired base two circularly polarized polarization states: $\hat{\mathbf{R}}=1 / \sqrt{2} \cdot[1, i]$ (righthanded) and $\hat{\mathbf{L}}=1 / \sqrt{2} \cdot[1,-i]$ (left-handed). Letter $i$ denotes an imaginary unit. Now the vector of the light $\mathbf{E}_{P}$ emerging from the polarizer and falling on the analyzer can be written as:

$$
\begin{aligned}
& \mathbf{E}_{P}=\left\langle\mathbf{E}_{P}, \hat{\mathbf{R}}\right\rangle \hat{\mathbf{R}}+\left\langle\mathbf{E}_{P}, \hat{\mathbf{L}}\right\rangle \hat{\mathbf{L}}= \\
& =\exp \left(-i \alpha_{P}\right) \hat{\mathbf{R}}+\exp \left(+i \alpha_{P}\right) \hat{\mathbf{L}}=\mathbf{E}_{P R}+\mathbf{E}_{P L}
\end{aligned}
$$

where $\mathbf{E}_{P R}$ and $\mathbf{E}_{P L}$ denote the right-handed and lefthanded components of vector, respectively. Inserting Eq. (7) into Eq. (5) one can obtain the following formula in this case: 
$\mathbf{E}_{\text {out }}=\left\{\exp \left[-i\left(\alpha_{A}-\alpha_{P}\right)\right]\right\} \mathbf{A}+\left\{\exp \left[+i\left(\alpha_{A}-\alpha_{P}\right)\right]\right\} \mathbf{A} \equiv$

$\equiv \mathbf{E}_{\text {outR }}+\mathbf{E}_{\text {outR }}$

where $\mathbf{E}_{\text {outR }}$ and $\mathbf{E}_{\text {outL }}$ denote the right-handed and lefthanded components of the $\mathbf{E}_{\text {out }}$, respectively. Thus we can conclude that the Malus Law equation can be obtained again as an effect of the interference of two linearly polarized waves with the same polarization states (represented by vector A ) - only this time the component waves have the same amplitudes but different phases (dependent on $\alpha_{P}$ and $\alpha_{A}$ difference). These phases (or rather their difference) can be interpreted as a geometric phase; it depends only on mutual orientation of the polarizer and analyser azimuth angles $\left(\alpha_{P}\right.$ and $\left.\alpha_{A}\right)$. Again, for clarity, the graphical representation of the $\mathbf{E}_{P}$ vector's decomposition as well as the projection of $\mathbf{E}_{P R}$ and $\mathbf{E}_{P L}$ components into vector $\mathbf{A}$ is presented in Fig. 3. Unfortunately, this graphical visualization is not as clear as in the previous case (see Fig. 2) due to the difficulties in presenting the circle vectors $\mathbf{E}_{P R}$ and $\mathbf{E}_{P L}$ (in fact, we can draw only their amplitudes and there is no simple way to present their mutual phases).

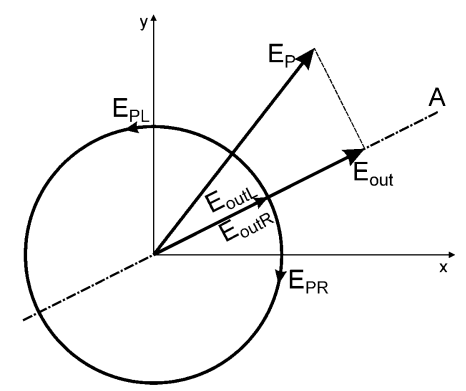

Fig. 3. The graphical representation of the $\mathbf{E}_{\mathrm{P}}$ vector's decomposition into right-handed $\left(\mathbf{E}_{\mathrm{PR}}\right)$ and left-handed $\left(\mathbf{E}_{\mathrm{PL}}\right)$ circular components as well as the projection of $\mathbf{E}_{\mathrm{PR}}$ and $\mathbf{E}_{\mathrm{PL}}$ components into vector $\mathbf{A}$ to

obtain the vector $\mathbf{E}_{\text {out }}$ of the light emerging from the analyzer.

The traditional approach assumes that the observed intensity changes of the light passing through a system of the linear polarizer and analyser with a variable angular orientation can be interpreted as an effect of the analyser's eigenwaves attenuation. Our new description assumes that we can explain the above-mentioned changes in light intensity using interferometry concepts. This approach means that we resigned from the eigenwave's description of the analyser's behaviour and instead proposed a decomposition of the incident light polarization state into two different new bases. The first base choice, linearly polarized, allows interpreting Malus Law as interference of two linearly polarized waves with the same polarization state, different amplitude and the same phase. The second decomposition, using circularly polarized vectors as a base, leads to the description in which Malus Law can be interpreted as interference of two waves with the same amplitude but different phase. This allows us to introduce the concept of the geometric phase into Malus Law. To sum up, we have described the analyzer as an element realizing the interference of two waves which are the components of the wave leaving the polarizer.

\section{References}

[1] P. Kurzynowski, W.A. Woźniak, M. Szarycz, J. Opt. Soc. Am. A 28, 475 (2011).

[2] W.A. Woźniak, P. Kurzynowski, M. Zdunek, Opt. Appl. Vol. XLIII, No. 2 (2013). 